\title{
相图热力学数据库及其计算软件: 过去、现在和将来
}

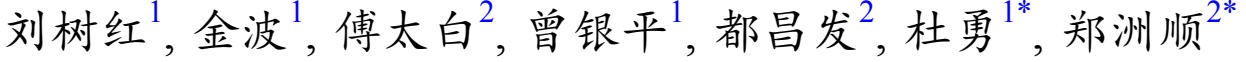 \\ 1. 中南大学轻质高强结构材料国防科技重点实验室, 长沙 410083 \\ 2. 中南大学数学与统计学院, 长沙 410083 \\ *通讯作者, E-mail: yong-du@csu.edu.cn; zszheng@csu.edu.cn
}

收稿日期：2018-12-11; 接受日期：2019-02-20; 网络版发表日期：2019-05-30

国家自然科学基金(编号: 51771235)和国家重点基础研究发展规划(编号: 2016YFB0701202)资助项目

摘要 相图热力学信息是材料设计和研发的重要基础之一. 基于热力学数据库的CALPHAD (CALculation of PHAse Diagram)方法使得多元多相材料的相图热力学计算成为可能. 随着材料基因组工程(Materials Genome Initiative, MGI)和集成计算材料工程(Integrated Computational Material Engineering, ICME)的发展, 各种材料的热力 学数据库及计算软件不断涌现. 本文结合本课题组在该方向的研究工作对相图热力学数据库及其计算软件的过 去进行了简单回顾, 并对现状以及未来的发展趋势进行了讨论.

关键词相图热力学数据库, 计算软件, CALPHAD方法, 材料基因组工程

\section{1 引言}

相图被誉为材料设计的指导书和冶金工作者的地 图, 其重要性不言而喻. 相图与热力学密切相关, 由相 图可以解析热力学信息，反过来基于热力学原理和热 力学数据也可以构筑相图. 随着热力学和计算机技术 的发展, 经过Gibbs ${ }^{[1]}$ 、van Laar ${ }^{[2,3]}$ 、Meijering ${ }^{[4,5]}$ 、 Kaufman $^{[6 \sim 8]} 、$ Kubaschewski $^{[9]}$ 、Hillert ${ }^{[10]}$ 和Ansara 等 ${ }^{[11]}$ 几代学者的共同努力, 20世纪70年代以来, 相图研 究从相平衡和热力学性质的实验测定进入到了相图和 热化学性质的计算机耦合的新阶段并逐渐发展成为一 种相图热力学研究的方法—CALPHAD (CALculation of PHAse Diagram或Computer Coupling of Phase Diagrams and Thermochemistry)方法.

CALPHAD方法基于热力学理论，建立具有一定
物理意义的Gibbs自由能模型来描述材料体系各组成 相的热力学性质，通过拟合由实验、第一性原理计算 以及经验公式获得的不同类型的数据来优化拟合模型 参数, 为多元多相材料构建工程精度的数据库. 人们利 用CALPHAD方法、通过Gibbs自由能建模和平衡计 算，将以往相对独立的相图信息和热力学信息统一起 来，为进一步描述材料相变和组织演化过程奠定了热 力学理论基础. 经过数十年的发展, CALPHAD方法在 材料科学研究和工程应用上受到越来越多的关注. 近 十年来，美、德等西方发达国家及我国相继实施了一 系列新材料发展计划 ${ }^{[12 ~ 17]}$, 旨在通过高通量的实验、 高通量计算和数据库的有机结合, 系统寻找材料组分/ 工艺-组织结构-性能的定量关系，从而加速新材料的 研发和应用速度. 2008年美国国家研究委员会在关于 增强未来国家竞争和国防安全的报告中指出( w w w .

引用格式: Liu S, Jin B, Fu T, Zeng Y, Du C, Du Y, Zheng Z. Thermodynamic databases and software: past, present and future. Sci Sin Chim, 2019, 49: 966-977, doi: $10.1360 / \mathrm{N} 032018-00258$ 
nap.edu/catalog/12199.html), 在实现集成计算材料工程 (ICME)的过程中, CALPHAD方法无疑是最重要的, 也 可能是目前唯一的通用工具. 美国西北大学的Olson教 授 ${ }^{[16,17]}$ 指出“尽管当前对于 “什么是材料基因”的讨论仍 在继续, 但可以确定我们现在已经拥有的“基因” 是 CALPHAD数据库及相关软件......”. 如图1所示, 基于 CALPHAD方法构筑的热力学和动力学以及热物性质 数据库是微结构模拟与性能预测的基本输入参数来 源. CALPHAD方法成功的两大要素是精确地描述材 料体系的相图热力学数据库和先进的综合性计算软 件. 本文从相图热力学数据库及其软件两个方面, 简述 了相关研究进展.

\section{2 相图热力学数据库}

正如热力学是进行一切材料研究的基础, 精确的 相图热力学数据库自然也是进行相平衡和热力学计算 的基础, 是进行材料相变模拟和组织演化过程模拟的 关键输入参数. 在CALPHAD相图热力学数据库中存 储的并不是一些带有标记的、相互关联的数据的集 合, 而是所研究的材料体系中各个相的Gibbs自由能模 型中定义的参数值或表达式. 由于Gibbs自由能包含了 成分、温度、压强变量, 所以相图热力学数据库可以 准确地表述宽广成分和温度、压力范围内的热力学性 质的变化. 数据库通常以纯文本格式存储, 文件比较 小, 即使一个包含几十种元素的多元体系数据库, 其 文件也通常仅有1 2 MB.

CALPHAD数据库目前已在钢铁、铝基、镁基、 镍基、钛基合金、硬质合金、焊料、贵金属、陶瓷、 矿渣、水溶液等领域，甚至只有14年历史的高熵合金 的开发中得到了广泛应用，相信未来还会应用于更广 阔的领域或环境. 构建数据库是个繁杂的过程, 需要 大量实验数据的支持, 还需要设计专门的实验和实验 方法. 根据CALPHAD方法构建数据库的要求, 在建立 多组元数据库之前必须优化低组元子体系数据. 例如, 要建立一个五元体系A-B-C-D-E的热力学数据库, 需 要从纯组元A、B、C、D、E开始, 研究五个纯组元的 晶格稳定性参数的准确性; 在此基础上, 建立 10 个二元 体系A-B、A-C、A-D、A-E、B-C、B-D、B-E、CD、C-E、D-E的热力学描述; 然后是 10 个三元系A-BC、A-B-D、A-B-E、A-C-D、A-C-E、A-D-E、B-C-

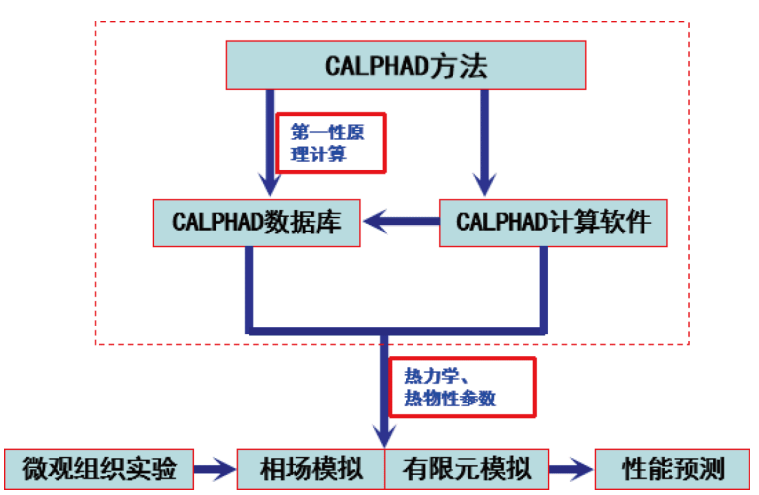

图 1 基于CALPHAD方法的微结构模拟与性能预测(网络 版彩图)

Figure 1 Microstructure simulation and performance prediction based on CALPHAD method (color online).

D、B-C-E、B-D-E及C-D-E; 接着是 5 个四元体系A-BC-D、A-B-C-E、A-B-D-E、A-C-D-E、B-C-D-E的热 力学描述及最终五元A-B-C-D-E体系的相图热力学数 据库的构筑. 由于过程的繁杂性, 并不是所有的子体系 都有相应的热力学描述. 通常的数据库是针对某一特 定类型的合金或特殊应用. 以铝合金为例, 要建立的 多元铝合金相图热力学数据库通常要包含绝大多数二 元体系的相图热力学描述、大部分含 $\mathrm{Al}$ 的三元体系的 热力学描述和少数关键四元体系富 $\mathrm{Al}$ 角的热力学描 述. 然而, 近年来兴起的高熵合金由于含有多个主要元 素且一般应用在等原子比成分附近, 相应的热力学数 据库的开发因此变得非常困难. 原则上讲, 所有的二 元、三元以及四元体系都很重要. 四元体系的热力学 描述大多由子体系外推而来, 其评估与优化一般侧重 于调节其子二元和三元体系的描述. 一般而言, 一个 数据库包含的具有可靠的相图热力学描述的三元系的 数量可作为数据库准确度的一个评判标准. 故实验方 面需要设计二元、三元体系热力学性质的精确测定, 实验样品必须采用高纯度原材料. 为了保证数据的可 靠性和自洽性, 还往往需要设计材料服役温度、压力 区间之外的实验 ${ }^{[18]}$.

\section{1 第一代相图热力学数据库(过去)}

从1959年开始, Kaufman ${ }^{[7]}$ 系统研究了晶格稳定性 (lattice stability, 即纯元素的稳定结构与其他亚稳或不 稳定结构之间的Gibbs自由能之差, 反映各种结构的相 对稳定性). 晶格稳定性概念的提出使得研究合金体系 不同相间平衡相界的计算变得简单易行, 为其后热力 
学计算方法成功地向二元及多元体系扩展奠定了基 础. 可以说, 如果没有晶格稳定性的提出, 就没有后来 多元相图热力学数据库的建立. 在此基础上, Kaufman 等 ${ }^{[8]}$ 率先采用计算机系统计算了大量二元合金体系和 一些三元合金体系的相图. 图2所示为其专著中用到的 过渡金属元素hcp_A3 和bcc_A2两种结构间的焓变和 熵变值 ${ }^{[8]}$.

其后，随着20世纪80年代通用热力学计算软件的 涌现, 复杂二元及多元体系的计算也成为可能. 这个 时期基于Kaufman纯组元晶格稳定参数构建的相图热 力学数据库, 称之为第一代相图热力学数据库.

\section{2 第二代相图热力学数据库(现在)}

晶格稳定参数(即纯组元热力学数据库)的合理性, 直接影响着后续二元、三元乃至多元数据库的构 筑 ${ }^{[19]}$. 为了能把各二元、三元体等子体系的热力学参 数合并到多组元热力学数据库中, 这些子体系的纯组 元信息必须一致. SGTE (Scientific Group Thermodata Europe)率先开展了这部分工作, 基于实验测定数据, 包括量热法、电化学方法、相图测定或耦合热力学计 算, 从大量的二元系、三元体系的外推, 从而为国际同 行提供一个纯组元标准 ${ }^{[19]}$. SGTE后续工作的更新, 已 被许多学术网站所收录 ${ }^{[20]}$.

基于SGTE纯组元晶格稳定性参数, 大量的二元、 三元乃至多元体系的热力学评估大量涌现. 这就为第 二代热力学数据库的构建创造了客观条件.

随着基于密度泛函理论(DFT)的第一性原理计算 方法的普及，一些学者计算了大量元素在 $0 \mathrm{~K}$ 时不同晶 体结构的晶格稳定性 ${ }^{[21,22]}$. 此外, 对于亚稳相的热力学 性质, 第一性原理计算也是唯一有效的计算工具. 由此 第一性原理辅助CALPHAD方法构筑相图热力学数据 库也越来越普及. 目前各大商用软件公司纷纷开发了 各种面向工程用材料体系的相图热力学数据库. 以铝 合金为例, 由于其作为轻质结构材料在航空航天、交 通运输及民用建筑领域的广泛应用, 各大商用软件公

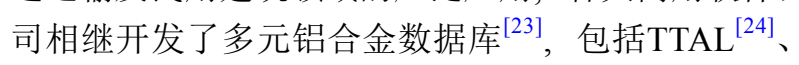
$\operatorname{Pan} \mathrm{AL}^{[25]}$ 及 $\mathrm{TCAL}^{[26]}$, 其中TCAL是中南大学杜勇、刘 树红等与瑞典Thermo-Calc公司合作开发. 从2011年5 月最初发布 $\mathrm{TCAL}^{[27]}$ 到Thermo-Calc公司现在扩展的 TCAL5，目前已包含35种元素 $(A 1 、 A g 、 B 、 B e 、 B i 、$ C、Ca、Cd、Ce、Co、Cr、Cu、Er、Fe、Ga、Ge、

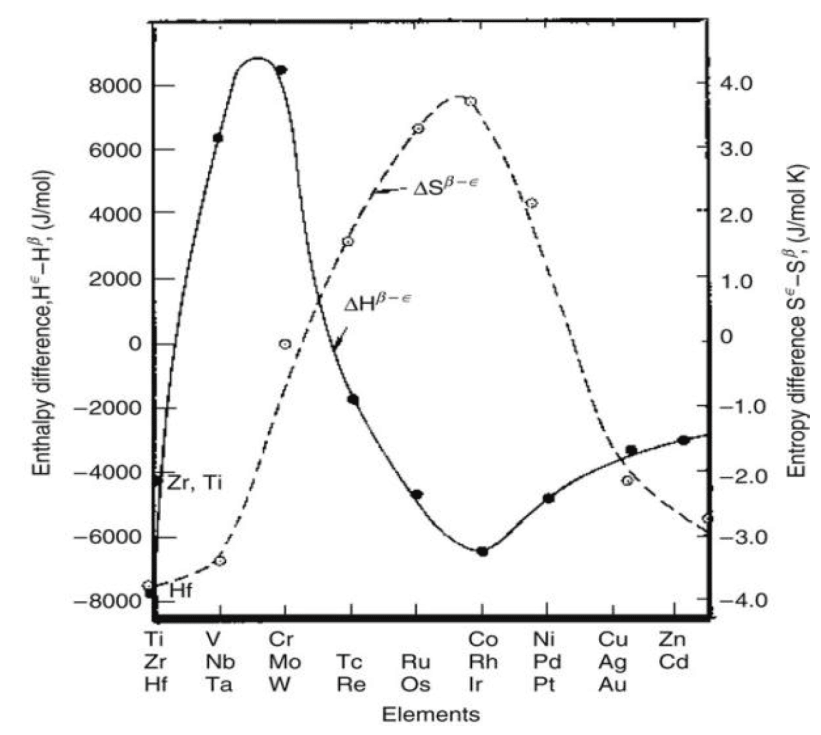

图 2 过渡元素hcp_A3 和bcc_A2结构间的焓变和熵变值 ${ }^{[8]}$ Figure 2 Enthalpy and entropy differences between the hcp_A3 and bcc_A2 forms of transition metals [8].

$\mathrm{H} 、 \mathrm{Hf} 、 \mathrm{In} 、 \mathrm{~K} 、 \mathrm{La} 、 \mathrm{Li} 、 \mathrm{Mg} 、 \mathrm{Mn} 、 \mathrm{Na} 、 \mathrm{Ni} 、 \mathrm{~Pb}$ 、 $\mathrm{Sc} 、 \mathrm{Si} 、 \mathrm{Sn} 、 \mathrm{Sr} 、 \mathrm{Ti} 、 \mathrm{~V} 、 \mathrm{Zn}$ 及 $\mathrm{Zr}$ ), 覆盖全成分范围的 258 个二元系和 87 个三元系以及 12 个含铝的四元体系 富铝角的热力学评估, 除了包含所评估二元、三元体 系的所有稳定相外, 还包含铝合金在铸造和时效过程 中起重要作用的亚稳相的热力学描述 ${ }^{[26]}$, 是目前国际 上包含元素和相最多的多元铝合金热力学数据库 ${ }^{[23]}$.

一般来说, 高精度的热力学数据库基于合理的热 力学模型和精确可靠的相图及热力学数据. TCAL的 构筑综合采用了实验测定、相图计算(CALPHAD方 法)及第一性原理计算辅助的方法 ${ }^{[26,27]}$. 为了提高数据 库对商用合金预测的准确度, 还要充分考虑关键体系 的计算精度及商用合金的重要析出相, 并对这些体系 及析出相的热力学模型和参数进行相应调整 ${ }^{[26]}$. 例如, 要提高数据库对 $7 X X X$ 合金的预测准确度, 需要对 $\mathrm{Al}-$ $\mathrm{Zn}-\mathrm{Mg}-\mathrm{Cu}-\mathrm{Fe}$ 体系进行系统研究, 对构成该五元系的 重要三元体系的热力学参数进行调整, 并充分考虑 $\mathrm{S}^{\prime}-\mathrm{Al}_{2} \mathrm{CuMg} 、 \mathrm{~T}^{\prime}-\mathrm{Al}_{0.3} \mathrm{Mg}_{0.4} \mathrm{Zn}_{0.3}$ 及 $\eta^{\prime}-\mathrm{Al}_{3} \mathrm{Mg}_{2.5} \mathrm{Zn}_{3.5}$ 等亚 稳相的热力学描述 ${ }^{[28]}$. 图 3 是基于数据库计算的 $\mathrm{Al}-\mathrm{Cu}$ 稳定相图从 0 到 $50 \mathrm{at} \% \mathrm{Cu}$ 的区域. 相对于稳定相图显 示的富铝角较简单的相关系, 在商用 $2 X X X 、 6 \mathrm{XXX}$ 及 $7 \mathrm{XXX}$ 合金中还报道了一系列亚稳析出相及相应的析 出序列GPI区 $\rightarrow$ GPII区 $\left(\theta^{\prime \prime}\right) \rightarrow \theta^{\prime} \rightarrow \theta \rightarrow \Omega^{[29]}$. 沿着Mur$\mathrm{ray}^{[30]}$ 的工作, Chen等 ${ }^{[28]}$ 在 $\mathrm{Al}-\mathrm{Cu}$ 体系亚稳相的模拟工 
作中将GPI区处理成(A1)固溶体溶解度间隙的第二个 成分, 同时对Fcc_A1相的热力学参数进行调整. GPII 区处理成化学计量型 $\mathrm{Al}_{3} \mathrm{Cu}$ 相(也就是 $\theta^{\prime \prime}$ ). $\theta^{\prime}$ 是比 $\mathrm{GP}$ 区 热力学稳定性更高的相, 具有b.c.t. 结构 $(I-4 m 2, \mathrm{tP} 6)$, 与 $\alpha(\mathrm{Al})$ 在 $\{100\}$ 面呈半共格关系, 是主要的强化相. $\Omega$ 与稳定的 $\theta$ 相密切相关, 稳定的 $\theta-\mathrm{Al}_{2} \mathrm{Cu}$ 相具有 $\mathrm{C} 16$ 结 构, 与基体非共格. 图3也包含了利用数据库计算的AlCu体系Fcc_A1相对各种亚稳相析出的固溶度线.

为了了解多种合金元素对铝合金相形成及形变的 协同作用, 评估的二元、三元体系需要外推到四元体 系并结合有限的实验信息对外推结果进行验证. Al$\mathrm{Cu}-\mathrm{Mg}-\mathrm{Si}$ 是6XXX商用合金的基础体系. 在这个体系中 包含一些重要的中间相, 如 $\mathrm{Al}_{2} \mathrm{Cu} 、 \mathrm{Mg}_{2} \mathrm{Si} 、 \mathrm{~S}-\mathrm{Al}_{2} \mathrm{CuMg}$ 及四元化合物 $\mathrm{Q}$. 由于文献中对 $\mathrm{Q}$ 稳定存在的成分范围 存在争议, 目前TCAL5数据库中, $\mathrm{Q}$ 被处理成 $\mathrm{Al}_{5} \mathrm{Cu}_{2} \mathrm{Mg}_{8}$ $\mathrm{Si}_{6}$ 的线性化合物. 图4是利用TCAL5数据库计算的Al$\mathrm{Cu}-\mathrm{Mg}-\mathrm{Si}$ 体系在含 $4.5 \mathrm{wt} \% \mathrm{Cu}$ 的成分和在 $773 \mathrm{~K}$ 温度 下的局部等温截面. 图5是该体系在含 $0.6 \mathrm{wt} \% \mathrm{Si}$ 的成 分和在 $773 \mathrm{~K}$ 温度下的局部等温截面. 其富 $\mathrm{Al}$ 角给出了 几个 $6 \mathrm{XXX}$ 系列工业铝合金的成分范围.

\section{3 第三代相图热力学数据库(将来)}

现在广泛应用的SGTE纯组元晶格稳定性参数基 于热容的温度多项式表达形式, 其系数没有物理意义, 描述的温度范围也限于 $298.15 \mathrm{~K}$ 以上, 所以第二代数 据库不能描述室温以下的相图和热力学性质. 此外, 固态熔点以上和液态凝固点以下的热容描述具有弊 端. 针对这些问题, 一些研究组自 1995年开始着力开发 新的能表述整个温度范围的具有物理意义的纯组元热 容模型 ${ }^{[31 ~ 34]}$, 并对二元、三元体系进行了重新热力学 优化, 以期获得研究体系在整个温度范围的热力学描 述. 毫无疑问, 这是一个浩大的工程, 需要全球CALPHAD工作者的合作和努力. 目前对于二元、三元体 系的研究仍相当有限 ${ }^{[35]}$.

此外, 随着现代制备加工技术的发展, 越来越多的 学者关注各种外场对材料制备、加工过程组织结构和 性能的影响 ${ }^{[36]}$. 自然地, 未来的第三代相图热力学数 据库应该是现有相图热力学数据库的进一步细化和升 华, 应充分考虑外场变量对热力学性质的影响. 常见的 外场变量有温度、压力、电场、磁场等, 其中外加磁 场与传统能量场(温度场和压力场)的作用机理不同,

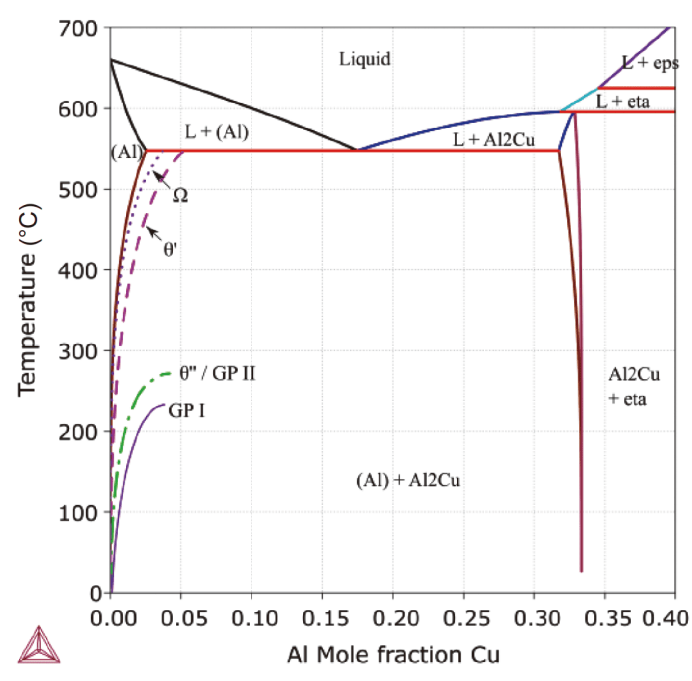

图 3 基于 $\mathrm{TCAL}$ 数据库计算的 $\mathrm{Al}-\mathrm{Cu}$ 稳定相图以及 $\mathrm{Fcc}$ - $\mathrm{A} 1$ 相对各种亚稳析出相的固溶度线 ${ }^{[26]}$ (网络版彩图)

Figure 3 Calculated Al-Cu stable phase diagram, along with Fcc_A1 solvi relative to all metastable Al-Cu precipitates [26] (color online).

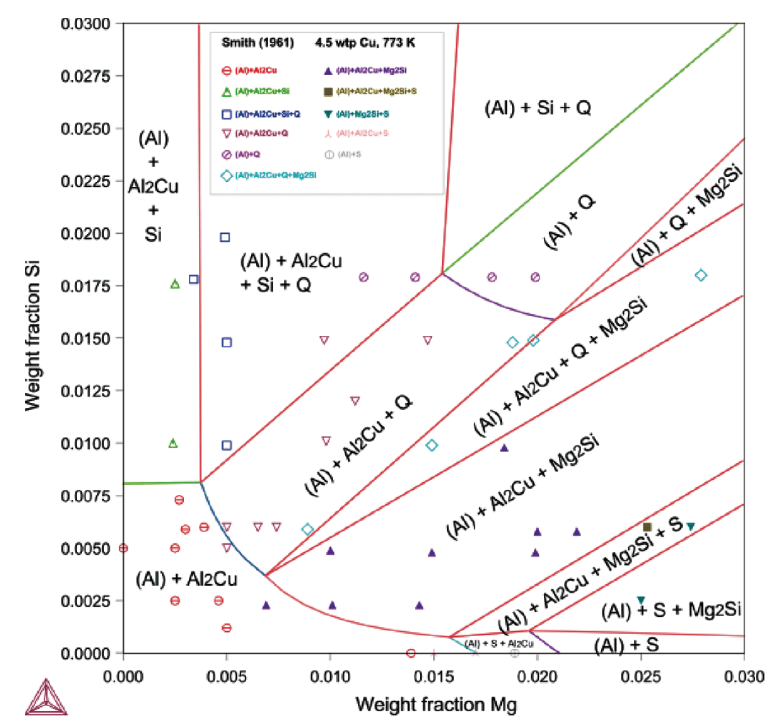

图 4 基于 TCAL5 计算的 $773 \mathrm{~K} \mathrm{Al-4.5} \mathrm{wt} \% \mathrm{Cu}-\mathrm{Mg}-\mathrm{Si}$ 的富 $\mathrm{Al}$ 角等温截面(网络版彩图)

Figure 4 Calculated Al-Cu-Mg-Si isothermal sections at $4.5 \mathrm{wt} \% \mathrm{Cu}$ at $773 \mathrm{~K}$ (color online).

它是通过影响物质中电子的运动状态使各相的内能发 生变化, 从而对相平衡产生影响. 在磁场下, 物质的电 子由于受到磁场的作用而使其自旋发生变化从而改变 了相的能量, 其降低量取决于各相的磁化强度或磁化 率. 磁化强度(或磁化率)越高的相, 其Gibbs自由能就 越低, 相就会变得越稳定. 因此温度场与磁场共同作 用可以使材料中固态相的相对稳定性发生变化, 从而 


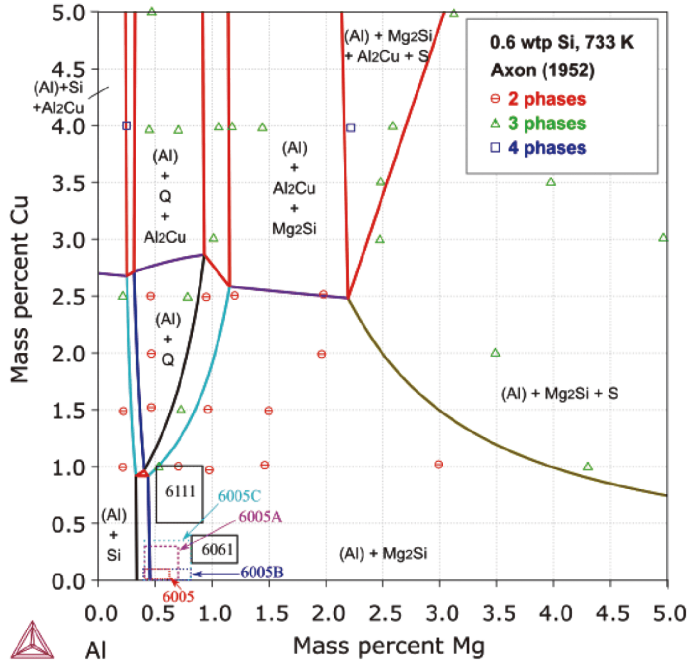

图 5 基于TCAL5 计算的Al-Cu-Mg- $0.6 \mathrm{wt} \% \mathrm{Si}$ 在 $773 \mathrm{~K}$ 的富 $\mathrm{Al}$ 角等温截面(网络版彩图)

Figure 5 Calculated $\mathrm{Al}-\mathrm{Cu}-\mathrm{Mg}-\mathrm{Si}$ isothermal sections at $0.6 \mathrm{wt} \% \mathrm{Si}$ at $733 \mathrm{~K}$ (color online).

改变相变路径.

基于热力学定律, 磁性相的外磁场磁性自由能为

$G_{\mathrm{m}}^{e x}=-\int_{0}^{B} M \mathrm{~d} B$,

其中 $M$ 为磁化强度, $B$ 为磁感应强度. 已知 $M-B$ 的关系 就可以求得外磁场导致的自由能. 目前, 基于局域理论 的Weiss分子场模型 ${ }^{[37]}$ 是较成熟的计算模型, 借助于上 述模型可计算物质的磁化强度和磁化率随温度变化和 磁场变化的规律. 最近，中南大学的杜勇 ${ }^{[38]}$ 基于该模 型计算了不同外磁场作用下Bi-Mn二元相图，并与实 验数据 ${ }^{[39-45]}$ 进行比较, 如图6所示. 随着外磁场强度的

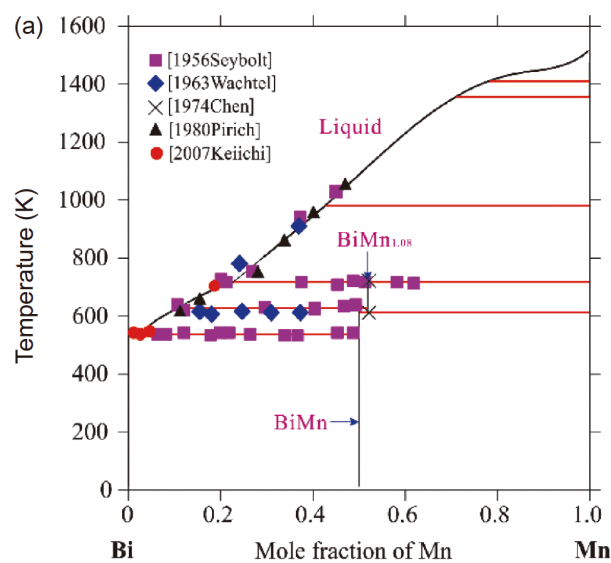

增加, 铁磁相BiMn更加稳定, 顺磁相BiMn $\mathrm{Bn}_{1.08}$ 的稳定温 度范围变小. 15 和 $18 \mathrm{~T}$ 磁场下的相图变化不大. 当外磁 场为 $50 \mathrm{~T}$ 时, 顺磁相 $\mathrm{BiMn}_{1.08}$ 将不再稳定, 铁磁相的工 作温度将大大提高. 从本工作计算的结果与实验数据 对比, 发现该磁场能量模型能准确描述Bi-Mn二元系, 有望应用于其他体系并推广到多元系.

除了外磁场的作用，高压及极限温度的作用也是 第三代相图热力学数据库关注的热点. 第二代相图热 力学数据库描述的凝聚态相最初大多是在常压下的情 况. 在常压下, 压力对凝聚态相的Gibbs自由能贡献很 小 ${ }^{[46]}$, 通常可以忽略不计. 但高压对Gibbs自由能的贡 献将不能被忽略. 一些材料的制备、加工及服役条件 与压力密切相关, 因此构建这些材料体系的高压相图 热力学数据就显得十分必要. 目前, 许多第二代的商 业热力学数据库完成了升级, 包含有完整的摩尔体积 与热膨胀参数. 最近, 北京科技大学的李长荣教授团 队 ${ }^{[47]}$ 采用CALPHAD方法研究了一些纯金属元素不同 结构的Gibbs自由能随温度和压强的表达式，并绘制了 一系列热力学性质随温度和压强的变化曲线. 图7是该 团队计算的温度和压强对 $\mathrm{Mg}$ 和 $\mathrm{Zr}$ 的各种凝固态结构 的压缩能的影响. 由计算结果可知，当压强高于 $10^{9} \mathrm{~Pa}$ 时，其对吉布斯能的贡献将不能被忽略. 中南大学的 Huang等 ${ }^{[48]}$ 最近也尝试构筑了多元铝合金摩尔体积数 据库, 图8为他们通过构筑纯组元的摩尔体积数据库计 算的纯镁的 $P-T$ 相图与文献数据的比较.

除了上述提到的外场作用，尺寸效应也是第三代 相图热力学数据库应该要关注的热点. 纳米结构材料 是一种相对于传统块体结构材料的新型结构材料, 相

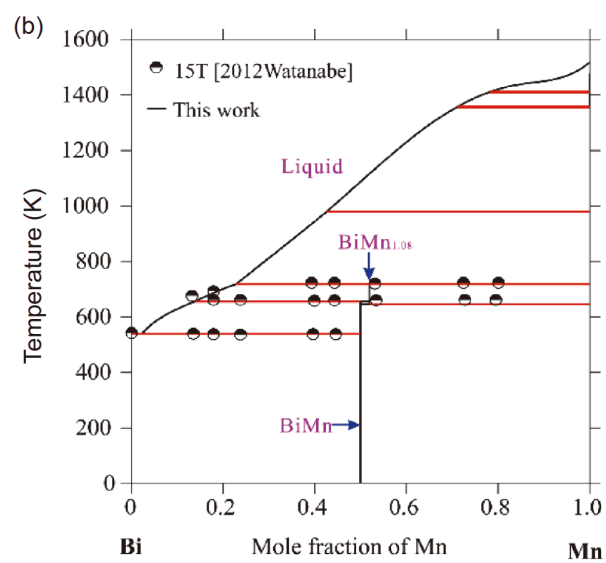

图 6 计算的不同磁场作用下的Bi-Mn相图与实验数据的比较. (a) $0 \mathrm{~T}$; (b) $15 \mathrm{~T}$ (网络版彩图)

Figure 6 Calculated phase diagram of Bi-Mn at different magnetic field with experimental data. (a) $0 \mathrm{~T}$; (b) $15 \mathrm{~T}$ (color online). 

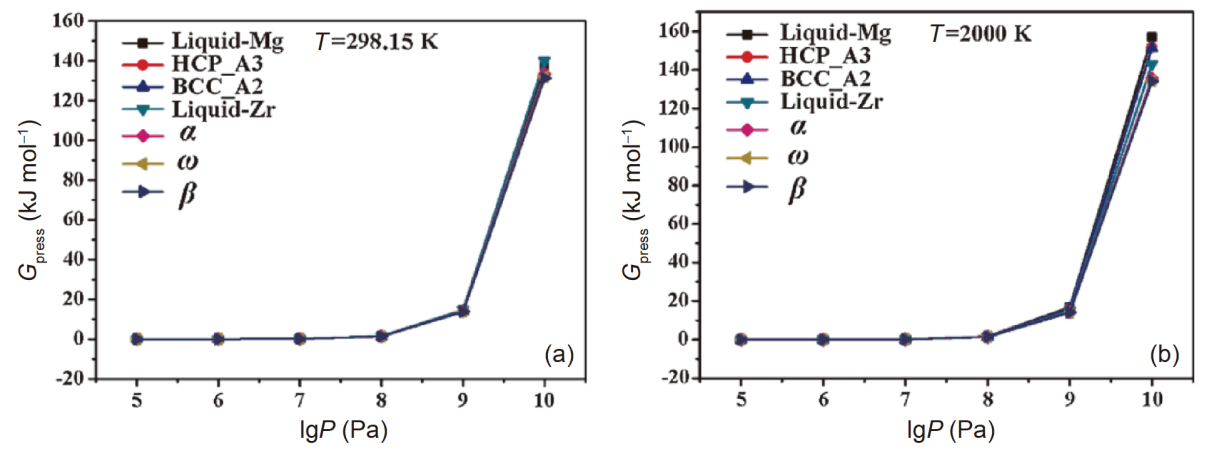

图 7 计算的 $G_{\text {press }}-\lg P$ 的关系曲线. (a) $298.15 \mathrm{~K}$; (b) $2000 \mathrm{~K}^{[47]}$ (网络版彩图)

Figure 7 The $G_{\text {press }}-\lg P$ relationship of Mg and Zr. (a) $T=298.15 \mathrm{~K}$; (b) $T=2000 \mathrm{~K}$ [47] (color online).

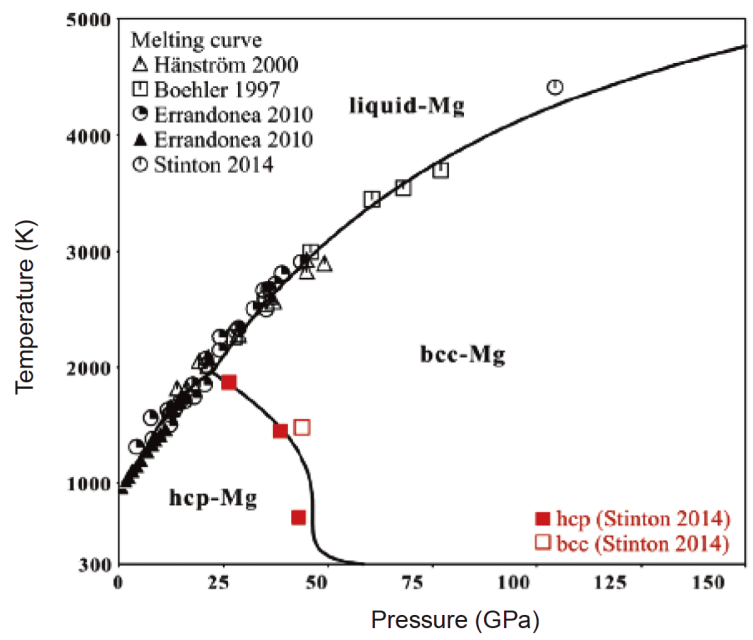

图 8 基于摩尔体积数据库计算的纯镁的 $P-T$ 图 ${ }^{[48]}$ (网络版 彩图)

Figure 8 Calculated $P$-T phase diagram of pure $\mathrm{Mg}[48]$ (color online).

对于制备、工艺实验研究的突飞猛进发展，目前关于 热力学基础理论的研究还不够完善. 例如, 纳米尺寸 材料的热力学稳定性问题，更没有CALPHAD类型多 元体系相图热力学数据库构筑的系统研究报道. 纳米 材料的热力学性质都存在尺寸效应. 吉林大学的蒋青 教授团队 ${ }^{[49,50]}$ 在纳米材料界面能的尺寸效应方面做了 大量的研究工作. 图9为计算的不同结构纳米碳转变的 临界尺寸随温度的变化与文献数据的比较 ${ }^{[49]}$.

Tanaka和Hara ${ }^{[51,52]}$ 将表面能引入到CALPHAD热 力学模型中, 开启了CALPHAD方法预测纳米颗粒的 热力学稳定性和热力学性质的新篇章. 北京科技大学 的Qiao 等 ${ }^{[53]}$ 详细介绍了表面张力的实验测定和计算方 法, 特别是 $\beta$ 参数的获取方法. 韩国高丽大学的Lee
等 ${ }^{[54,55]}$ 推导了纯组元化学势和过剩吉布斯自由能为目 前商用热力学计算软件可接受的格式, 并绘制了 Ag$\mathrm{Au} 、 \mathrm{Au}-\mathrm{Cu}$ 二元纳米相图. 此后的许多工作大多围绕 纳米相表面能的推导与计算展开. 最近匈牙利密什科 尔茨大学的Kaptay教授 ${ }^{\left[{ }^{[6]}\right.}$ 通过引入比表面积 $A_{\mathrm{sp}, \mathrm{o}}$ 推导 关系获得纳米相的摩尔吉布斯自由能与比表面积 $A_{\mathrm{sp}, \varphi}$ 呈正比, 与尺寸呈反比. 当尺寸大于 $100 \mathrm{~nm}$ 时, 尺寸效 应不再明显.

$G_{\mathrm{m}, \varphi} \cong G_{\mathrm{m}, \mathrm{b}, \varphi}+A_{\mathrm{sp}, \varphi} \times V_{\mathrm{m}, \varphi} \times \sigma_{\varphi}$,

其中 $V_{\mathrm{m}, \varphi}$ 为 $\Phi$ 相的的摩尔体积, $\sigma_{\varphi}$ 为 $\Phi$ 相的表面张力 (表 面能).

\section{3 相图热力学计算软件}

相图热力学数据库的构筑离不开相图热力学计算 软件的开发. 目前广泛使用的的相图热力学软件按其 性质可分为: 通用相图热力学计算软件和开源相图热 力学计算软件.

\section{1 通用相图热力学计算软件}

由于相图的实验测定(尤其是多元体系)成本高昂 且耗时极长, Van $\operatorname{Laar}^{[2,3]}$ 早在1908年就提出了相图计 算这一观点. 他采用理想溶体模型和规则溶体模型计 算了大量具有不同拓扑特征的二元系相图, 阐明了二 元体系中相的热力学稳定性与相图特征之间的关系. 随后, Meijering ${ }^{[4,5]}$ 将Van Laar的工作拓展到更高组元, 同样采用了规则溶体模型计算了大量具有不同拓扑特 


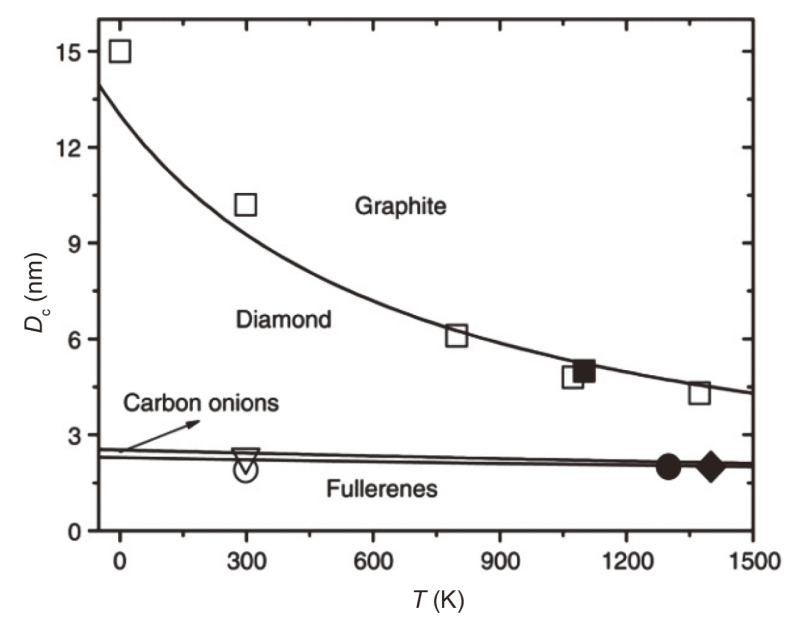

图 9 不同结构的纳米碳转变的临界尺寸对温度的变化与 文献数据的比较 ${ }^{[49]}$

Figure 9 Calculated $D_{\mathrm{c}}-T$ transition diagram of nanocarbon [49].

性的三元系相图. 这两位学者的研究工作在理解合金 相平衡与合金相的热力学稳定性的关系上做出了卓越 的贡献.

在Van Laar和Meijering关于相图计算的开创性工 作的基础上, Kaufman和Bernstein ${ }^{[8]}$ 提出并发展了与计 算机处理功能相结合的相图计算技术(CALPHAD). 其 后，一系列通用相图计算软件应运而生，包括ChemSage ${ }^{[57,58]} 、$ FactSage ${ }^{[59,60]}$ 、Lukas程序 ${ }^{[61]} 、$ MatCalc $^{[62]} 、$ MTDATA $^{[63,64]} 、$ Pandat $^{[65 \sim 67]} 、$ ThermoCalc ${ }^{[68 \sim 70]}$ 等. 特别值得一提的是 Thermo-Calc 可以计算 各种各样条件下的相平衡, 并拥有强大的相图优化模 块Parrot ${ }^{[68,71]}$. 为优化获得现今所存在的大多数热力学 参数和数据库做出了卓越的贡献.

早期相图热力学计算软件采用的是局域优化算 法, 稳定相图的计算依赖于合适的初始值, 计算结果 可能是亚稳平衡 ${ }^{[72]}$. 如图10所示, 某一 $T 、 P$ 时， $\alpha$ 和 $\beta$ 相的吉布斯自由能随成分的变化关系. 由 $\beta$ 相的吉布 斯自由能曲线可知，该相存在相分离，即分解成相同 晶体结构的 $\beta^{\prime} 、 \beta^{\prime /}$ 两相. 这时候可能存在三种两相平 衡，即 $\alpha+\beta^{\prime} 、 \alpha+\beta^{\prime \prime}$ 及 $\beta^{\prime}+\beta^{\prime \prime}$ ，但只有 $\alpha+\beta^{\prime \prime}$ 是稳定平衡. 在这种情况下, 早期软件的计算只有用户提供合适初 始值时才能获得稳定相图，否则可能会得到亚稳 相图.

为了避免获得稳定相图的不确定性问题, Pandat 率先采用了全局自由能最小化算法 ${ }^{[65]}$, 用户不用设定
初始值就可以实现多元体系稳定相图的计算. 然而很 多情况下同平衡计算相比，亚稳相平衡同样或甚至更 加重要, 因此Thermo-Calc现设有全局或局部自由能最 小化两个计算选项 ${ }^{[70]}$.

另外，为了方便用户使用，一些软件开发出多界 面、多系统模式. 例如, Thermo-Calc包含传统的交互 指令模式和用户友好的图形界面模式, 并可以在MS Windows、Linux以及Mac OS系统上运行 ${ }^{[70]}$. 另一方 面软件集成度更高, 除了能完成热力学相平衡计算和 扩散相变动力学模拟, 还集成了时效析出模拟模 块 ${ }^{[66,73 \sim 75]}$. 近期发行的 Thermo-Calc还包括材料性质模 型模块, 可用来计算界面能、析出相粗化速率以及钢 中的马氏体和珠光体转变等.

在集成计算材料工程(ICME)的环境中, 各种计算 材料学软件之间的便利对接至关重要. 因此不少相图 热力学计算软件都提供有关的应用程序接口(API)或 软件开发程序包(SDK). 例如, ChemSage和FactSage用 户可以使用ChemApp; Pandat用户可以调用PanEngin API; Thermo-Calc用户可以根据编程语言需要调用 Thermo-Calc SDK中的TQ (Fortran程序库)、TC-API (C程序库)、TC-Matlab Toolbox (Matlab程序库)和近 期推出的TC-Python (Python程序库). 尤其值得一提的 是, 由于Python的简洁性、易读性以及可扩展性, 非常 适合数据处理与图表制作，是目前最广泛使用的及人 工智能领域首选的科学计算计算机语言. TC-Python的 推出将为科研工作者应用CALPHAD数据库与软件带 来极大的便利.

以上介绍的通用相图热力学计算工具大多为商用 软件. 不过针对学术界Thermo-Calc提供免费的学术版 和配套的Hillert热力学教学材料 ${ }^{[70]}$. 这个特殊版本虽 然限于二元和三元系, 但可满足科研人员的相图优化 需求并方便材料热力学和动力学的教学.

\section{2 开源相图热力学计算软件}

如上所述, 使用大多数相图热力学计算软件需要 付费许可. 可以免费获得的学术版本也是基于商用版. 其体系架构均是封闭的, 研究人员不能够根据自己需 要植入新的热力学模型、测试新算法并拓展软件新功 能. 因此开源软件应运而生. 虽然开源程序有待进一步 成熟和完善, 同时在实践中需要编程经验和较长学习 


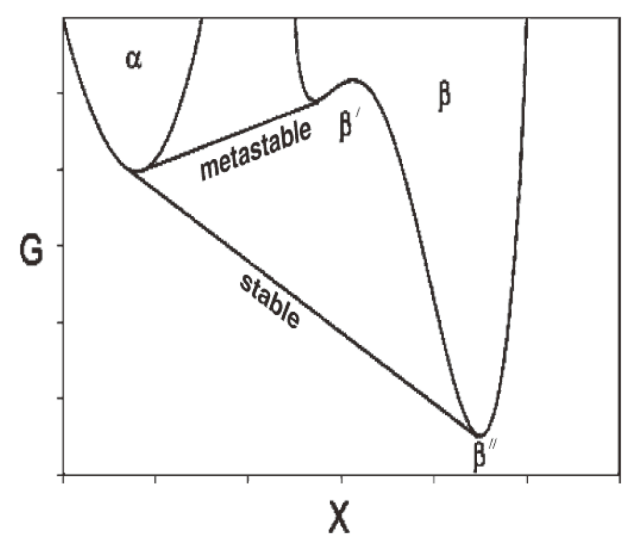

图 10 特定温度和压强时, $\alpha$ 和 $\beta$ 相的吉布斯自由能曲线, 显 示 $\beta$ 相存在相分离 ${ }^{[72]}$

Figure 10 Gibbs energies of $\alpha$ and $\beta$ as a function of composition at constant $T$ and $P$ with $\beta$ exhibiting phase separation [72].

时间，但是从理论上讲开源程序能够更高效地实现 ICME框架下各种计算方法的集成，包括来源于其他 方法的数据的利用. 长期以来DFT、相场模拟、有限 元模拟都有相应的开源程序. 最近, 开源相图热力学 计算软件OpenCalphad $(\mathrm{OC})^{[76]}$ 和PyCalphad ${ }^{[77]}$ 的出现 填补了原子尺度和介观尺度之间开源软件的空白. OC 自2015年首次发布以来，在模型和算法上不断进行改 进 ${ }^{[78]}$. 需要注意的是, 与所有的开源程序一样, $\mathrm{OC}$ 和 PyCalphad的使用者应了解并遵守其自带的开源协议.

相平衡计算的算法大多基于Gibbs自由能的最小 化, 最早由White等 ${ }^{[79]}$ 提出. OC中涉及的相图计算方法 主要由Eriksson和Rosen 等 ${ }^{[80]}$ 以及Hillert ${ }^{[10]}$ 发展而成. 1975年, Eriksson等 ${ }^{[80]}$ 将Gibbs自由能极小化生成的线 性方程组的求解分成两步来进行. 随后，在此基础上， Sundman等 ${ }^{[78]}$ 给出了详细的算法描述并对此进行了软 件实现.
目前，所有的软件都是通过迭代的方法来计算相 平衡，不同的迭代格式和迭代初值的选取可能会导致 诸如迭代结果发散、计算量庞大等问题，不同团队对 此做了相应的改进工作 ${ }^{[81 ~ 83]}$. 在Hillert ${ }^{[10]}$ 和Sundman 等 ${ }^{[78]}$ 所描述的算法中, 基于亚点阵模型并结合Lagrangian乘子方法和Taylor展开, 将非线性方程组转换为线 性方程组来求解, 这种方法被广泛地应用于各种软件 的热力学模型中. 随着亚点阵数目和组元数目的增加, 线性方程组系数矩阵的求逆的计算量将会变得越来越 大，从而很大程度上影响平衡计算的效率. 另一方面, 系数矩阵的条件数(用来描述矩阵计算对误差的敏感 度)一般也随着亚点阵数和组元数的增加而变大. 这 样, 在迭代求解的过程中, 由初值的选取和方程组的 求解而导致的误差, 可能会在计算组元分数的自然对 数时出现复数.

针对此种相图计算方法中, 由于求解线性方程组 而可能导致的计算量大和计算结果不稳定的情况, Fu 等 ${ }^{[84]}$ 对相图计算的求解过程进行了改进, 并且通过 MATLAB编程实现了在某些二元系中的应用, 验证了 新的求解方法可以在很大程度上减少线性方程组系数 矩阵的条件数且提高相图计算的效率.

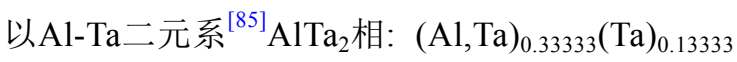
$(\mathrm{Al}, \mathrm{Ta})_{0.53334}$ 为例, $G_{M}$ 表示 $\mathrm{AlTa}_{2}$ 相摩尔 Gibbs自由能, $y_{1} 、 y_{2}$ 分别为第一个亚点阵中的元素 $\mathrm{Al} 、 \mathrm{Ta}$ 的组元分 数, $y_{3} 、 y_{4}$ 为第三个亚点阵中的元素 $\mathrm{Al} 、 \mathrm{Ta}$ 的组元分 数, $M_{\mathrm{Al}} 、 M_{\mathrm{Ta}}$ 分别表示单位结构式中元素 $\mathrm{Al} 、 \mathrm{Ta}$ 的摩 尔数, $\mu_{\mathrm{Al}} 、 \mu_{\mathrm{Ta}}$ 分别表示组元 $\mathrm{Al} 、 \mathrm{Ta}$ 在平衡共存的各相 中的化学势, $n$ 为 $\mathrm{AlTa}_{2}$ 相的相摩尔数, $\xi_{1}, \xi_{2}$ 是 $\mathrm{AlTa}_{2}$ 相 的Lagrange乘子, 这样, 求解各组元分数改变量的线性 系统为

$$
\left(\begin{array}{cccccc}
\frac{\partial^{2} G_{M}}{\partial^{2} y_{1}} & \frac{\partial^{2} G_{M}}{\partial y_{1} \partial y_{2}} & \frac{\partial^{2} G_{M}}{\partial y_{1} \partial y_{3}} & \frac{\partial^{2} G_{M}}{\partial y_{1} \partial y_{4}} & 1 & 0 \\
\frac{\partial^{2} G_{M}}{\partial y_{1} \partial y_{2}} & \frac{\partial^{2} G_{M}}{\partial^{2} y_{2}} & \frac{\partial^{2} G_{M}}{\partial y_{2} \partial y_{3}} & \frac{\partial^{2} G_{M}}{\partial y_{2} \partial y_{4}} & 1 & 0 \\
\frac{\partial^{2} G_{M}}{\partial y_{1} \partial y_{3}} & \frac{\partial^{2} G_{M}}{\partial y_{2} \partial y_{3}} & \frac{\partial^{2} G_{M}}{\partial^{2} y_{3}} & \frac{\partial^{2} G_{M}}{\partial y_{3} \partial y_{4}} & 0 & 1 \\
\frac{\partial^{2} G_{M}}{\partial y_{1} \partial y_{4}} & \frac{\partial^{2} G_{M}}{\partial y_{2} \partial y_{4}} & \frac{\partial^{2} G_{M}}{\partial y_{3} \partial y_{4}} & \frac{\partial^{2} G_{M}}{\partial^{2} y_{4}} & 0 & 1 \\
1 & 1 & 0 & 0 & 0 & 0 \\
0 & 0 & 1 & 1 & 0 & 0
\end{array}\right)\left(\begin{array}{c}
\Delta y_{1} \\
\Delta y_{2} \\
\Delta y_{3} \\
\Delta y_{4} \\
\frac{\xi_{1}}{n} \\
\frac{\xi_{2}}{n}
\end{array}\right)=\left(\begin{array}{c}
\frac{\partial M_{\mathrm{Al}}}{\partial y_{1}} \mu_{\mathrm{Al}}+\frac{\partial M_{\mathrm{Ta}}}{\partial y_{1}} \mu_{\mathrm{Ta}}+\frac{\partial G_{M}}{\partial y_{1}} \\
\frac{\partial M_{\mathrm{Al}}}{\partial y_{2}} \mu_{\mathrm{Al}}+\frac{\partial M_{\mathrm{Ta}}}{\partial y_{2}} \mu_{\mathrm{Ta}}+\frac{\partial G_{M}}{\partial y_{2}} \\
\frac{\partial M_{\mathrm{Al}}}{\partial y_{3}} \mu_{\mathrm{Al}}+\frac{\partial M_{\mathrm{Ta}}}{\partial y_{3}} \mu_{\mathrm{Ta}}+\frac{\partial G_{M}}{\partial y_{3}} \\
\frac{\partial M_{\mathrm{Al}}}{\partial y_{4}} \mu_{\mathrm{Al}}+\frac{\partial M_{\mathrm{Ta}}}{\partial y_{4}} \mu_{\mathrm{Ta}}+\frac{\partial G_{M}}{\partial y_{4}} \\
0 \\
0
\end{array}\right)
$$


上述六元线性系统可以简化为二元的等价线性系统:

$M\left(\begin{array}{c}\Delta y_{1} \\ \Delta y_{3}\end{array}\right)=\left(\begin{array}{l}\left(\frac{\partial M_{\mathrm{Al}}}{\partial y_{1}}-\frac{\partial M_{\mathrm{Al}}}{\partial y_{2}}\right) \mu_{\mathrm{Al}}+\left(\frac{\partial M_{\mathrm{Ta}}}{\partial y_{1}}-\frac{\partial M_{\mathrm{Ta}}}{\partial y_{2}}\right) \mu_{\mathrm{Ta}}-\left(\frac{\partial G_{M}}{\partial y_{1}}-\frac{\partial G_{M}}{\partial y_{2}}\right) \\ \left(\frac{\partial M_{\mathrm{Al}}}{\partial y_{3}}-\frac{\partial M_{\mathrm{Al}}}{\partial y_{4}}\right) \mu_{\mathrm{Al}}+\left(\frac{\partial M_{\mathrm{Ta}}}{\partial y_{3}}-\frac{\partial M_{\mathrm{Ta}}}{\partial y_{4}}\right) \mu_{\mathrm{Ta}}-\left(\frac{\partial G_{M}}{\partial y_{3}}-\frac{\partial G_{M}}{\partial y_{4}}\right)\end{array}\right)$

其中, 简化后的系数矩阵为

$M=\left(\begin{array}{cc}\frac{\partial^{2} G_{M}}{\partial y_{1}^{2}}-2 \frac{\partial^{2} G_{M}}{\partial y_{1} \partial y_{2}}+\frac{\partial^{2} G_{M}}{\partial y_{2}^{2}} & \frac{\partial^{2} G_{M}}{\partial y_{1} \partial y_{3}}-\frac{\partial^{2} G_{M}}{\partial y_{2} \partial y_{3}}-\frac{\partial^{2} G_{M}}{\partial y_{1} \partial y_{4}}+\frac{\partial^{2} G_{M}}{\partial y_{2} \partial y_{4}} \\ \frac{\partial^{2} G_{M}}{\partial y_{1} \partial y_{3}}-\frac{\partial^{2} G_{M}}{\partial y_{2} \partial y_{3}}-\frac{\partial^{2} G_{M}}{\partial y_{1} \partial y_{4}}+\frac{\partial^{2} G_{M}}{\partial y_{2} \partial y_{4}} & \frac{\partial^{2} G_{M}}{\partial y_{3}^{2}}-2 \frac{\partial^{2} G_{M}}{\partial y_{3} \partial y_{4}}+\frac{\partial^{2} G_{M}}{\partial y_{4}^{2}}\end{array}\right)$

针对这种简化过程, 现取温度 $T=1000 \mathrm{~K}$, 各组元分数 取为: $y_{1}=0.619111, y_{2}=0.380888 ; y_{3}=0.000258$, $y_{4}=0.999741$, 这组取值非常接近平衡状态时 $\mathrm{AlTa}_{2}$ 相的 各组元分数. 分别计算简化前后的线性系统, 得到其系 数矩阵的条件数由 $1.39445 \times 10^{12}$ 降为 1273.855 .

在MATLAB程序中, 仅将求解组元分数的线性系 统改为简化后的线性系统, 计算 $\mathrm{Al}-\mathrm{Ta}$ 二元相图(图11), 结果显示相图的计算时间可以由 $240 \mathrm{~s}$ 左右减少为 $24 \mathrm{~s}$ 左右.

此外，这种简化改进也适用于多元系的平衡计算. 同时随着在材料制造过程中电场、磁场等外场的引入 和人工智能以及机器学习的兴起, 如何在外场的条件 下设计合金成分以及利用人工智能篮选符合要求的合 金范围成为接下来相图热力学软件发展的热门方向.

\section{4 结论}

CALPHAD数据库及其计算软件是公认的当前 已经拥有的材料基因. 为了满足人们对新材料的设计 研发需求, 相图热力学方向研究人员应该对现有的相 图数据库进一步完善和细化, 构筑满足各个研究领域 需求的精确的相图热力学数据库. 外场作用(外磁 场、高压和极限温度等) 以及尺寸效应将是新一代相

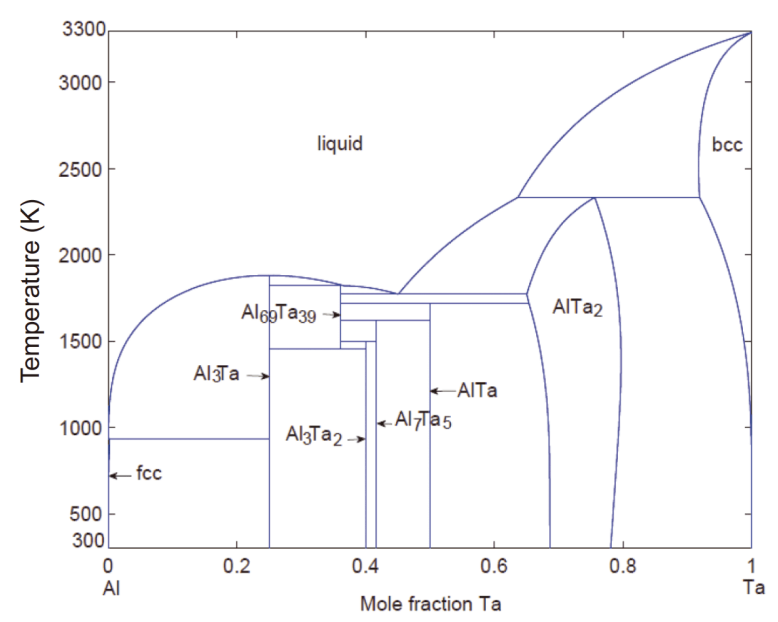

图 11 通过改进的算法结合MATLAB编程实现的Al-Ta相 图 ${ }^{[84]}$ (网络版彩图)

Figure 11 Calculated phase diagram of Al-Ta system through the improved algorithm combining with the MATLAB programming [84] (color online).

图热力学数据库的研究热点. 同时, 精确相图热力学 数据库的构筑离不开具有高效计算能力的相图热力 学计算软件的开发. 为了更高效地实现ICME框架下 各种计算方法的集成, 开发新的热力学模型, 测试新 算法并拓展软件新功能是现在相图热力学计算软件 的研究方向. 


\section{参考文献}

1 Gibbs JW. Trans Connecticut Acad, 1876, 2: 108-248

2 van Laar JJ. Z Phys Chem, 1908, 63: 216-253

3 van Laar JJ. Z Phys Chem, 1908, 64: 257-297

4 Meijering JL. Philips Res Rep, 1950, 5: 333-356

5 Meijering JL, Hardy HK. Acta Metall, 1956, 4: 249-256

6 Kaufman L, Cohen M. Trans AIME J Metals, 1956, 206: 1393-1401

7 Kaufman L. Acta Metall, 1959, 7: 575-587

8 Kaufman L, Bernstein H. Computer Calculation of Phase Diagrams. New York: Academic Press, 1970

9 Kubaschewski O, Alcock CB. Metallurgical Thermodynamic Chemistry. 5th ed. Oxford: Pergamon Press, 1979

10 Hillert M. Phys $B+C, 1981,103: 31-40$

11 Ansara I, Bernard C, Kaufman L, Spencer PG. Calphad, 1978, 2: 1-15

12 Gottstein G. Integral Materials Modeling: Towards Physics-Based Through-Process Models. Weinheim: Wiley-VCH Verlag GmbH \& Co. $\mathrm{KGaA}, 2007$. 17-32

13 Cicerone RJ, Charles MC. Integrated Computational Materials Engineering: A Transformational Discipline for Improved Competitiveness and National Security. Washington DC: The National Academies Press, 2008

14 Olson GB. Acta Mater, 2013, 61: 771-781

15 Yang R. Key Problems on Fundamental Science of Materials Design Based on Integrated Computation. 973 Project, 2011 (in Chinese) [杨锐. 基 于集成计算的材料设计基础科学问题. 973项目, 2011]

16 Olson GB. Scripta Mater, 2014, 70: 1-2

17 Olson GB, Kuehmann CJ. Scripta Mater, 2014, 70: 25-30

18 Lu X, Wang Z, Cui Y, Jin Z. Chin Sci Bull, 2013, 58: 3656-3664 (in Chinese) [鲁晓刚, 王卓, 崔予文, 金展鹏. 科学通报, 2013, 58: 3656-3664]

19 Dinsdale AT. Calphad, 1991, 15: 317-425

20 SGTE Unary Database version v4.4, 20 July 2001. E.g. see Elements and binary systems from Ag-Al to Au-Tl, in: Thermodynamic Properties of Inorganic Materials. Franke P, Neuschütz D, eds. Landolt-Börnstein: New Series Group 4: Phys Chem, vol. 19 XXVI Scientific Group Thermodata Europe (SGTE). Berlin: Springer, 2002

21 Wang Y, Curtarolo S, Jiang C, Arroyave R, Wang T, Ceder G, Chen LQ, Liu ZK. Calphad, 2004, 28: 79-90

22 Sluiter MHF. Calphad, 2006, 30: 357-366

23 Liu S, Ling D, Huang D, Zhang F, Du Y. Mater China, 2015, 34: 305-315 (in Chinese) [刘树红, 凌缔成, 黄丹丹, 张帆, 杜勇. 中国材料进展, 2015, 34: 305-315]

24 ThermoTech Al-based Alloys Database, TTAL8 (http://www.thermocalc.com/media/5985/ttal8.pdf)

PanAluminum, Thermodynamic database for multi-component aluminum-rich casting and wrought alloy (http://www.computherm.com/ download/database/PanA12017.pdf)

26 Chen HL, Chen Q, Engström A. Calphad, 2018, 62: 154-171

27 Du Y, Liu S, Zhang L, Xu H, Zhao D, Wang A, Zhou L. Calphad, 2011, 35: 427-445

28 Chen H, Chen Q, Bratberg J, Engström A. Mater Today Proc, 2015, 2: 4939-4948

29 Hutchinson CR, Fan X, Pennycook SJ, Shiflet GJ. Acta Mater, 2001, 49: 2827-2841

30 Murray JL. Phase Diagr Bin Copp Alloy, 1990: 18-42

31 Chase M, Ansara I, Dinsdale A, Eriksson G, Grimvall G, Hoglund L, Yokokawa H. Calphad, 1995, 19: 437-447

32 Chen Q, Sundman B. J Phase Equilib, 2001, 22: 631-644

33 Palumbo M, Burton B, Costa e Silva A, Fultz B, Grabowski B, Grimvall G, Hallstedt B, Hellman O, Lindahl B, Schneider A, Turchi PEA, Xiong W. Phys Status Solidi B, 2014, 251: 14-32

34 Roslyakova I, Sundman B, Dette H, Zhang L, Steinbach I. Calphad, 2016, 55: 165-180

35 Jiang Y, Zomorodpoosh S, Roslyakova I, Zhang L. Calphad, 2018, 62: 109-118 
Chen W, Qi J, Zhao T, Zhao Z, Wang J. Light Alloy Fabric Technol, 2013, 41: 15-19 (in Chinese) [陈文, 齐锦刚, 赵涛, 赵作福, 王建中. 轻合金 加工技术, 2013, 41: 15-19]

37 Jile D. Introduction to Magnetism and Magnetic Materials. Boca Raton: Chapman and Hall, 1991

Du Y. 19th National Thermochemisty and thermal analysis symposium by Chinese Chemical Society. Shenyang, 2018 (in Chinese) [杜勇. 中国化 学会第十九届全国化学热力学与热分析学术会议. 沈阳, 2018] Seybolt AU, Hansen H, Roberts BW. Trans Am Inst Min Metall Eng, 1956, 206: 606-610

Wachtel E, Damm R. Z Metallk, 1963, 54: 693-697 Royal Institute of Technology, Stockholm, 1984 
Sundman B, Lu XG, Ohtani H. Comput Mater Sci, 2015, 101: 127-137

White WB, Johnson SM, Dantzig GB. J Chem Phys, 1958, 28: 751-755

Eriksson G, Rosen E. Chem Scri, 1973, 19: 193-194

Emelianenko M, Liu ZK, Du Q. Comput Mater Sci, 2006, 35: 61-74

Piro MHA, Simunovic S, Besmann TM, Lewis BJ, Thompson WT. Comput Mater Sci, 2013, 67: 266-272

Snider J, Griva I, Sun X, Emelianenko M. Calphad, 2015, 48: 18-26

Fu T, Zheng Z, Du Y, Wang J, Du C, Jin B, Liu Y, Liu S. Comput Mater Sci, 2019, 159: 478-483

Du Y, Schmid-Fetzer R. J Phase Equilib, 1996, 17: 311-324

\title{
Thermodynamic databases and software: past, present and future
}

\author{
Shuhong Liu ${ }^{1}$, Bo Jin ${ }^{1}$, Taibai $\mathrm{Fu}^{2}$, Yinping Zeng ${ }^{1}$, Changfa Du ${ }^{2}$, Yong Du ${ }^{1 *}$, Zhoushun Zheng ${ }^{2 *}$ \\ ${ }^{1}$ National Key Laboratory of Science and Technology for National Defence on High-strength Structure Materials, Central South University, \\ Changsha 410083, China \\ ${ }^{2}$ School of Mathematics and Statistics, Central South University, Changsha 410083, China \\ *Corresponding authors (email: yong-du@csu.edu.cn; zszheng@csu.edu.cn)
}

\begin{abstract}
Phase diagram and thermodynamic data are essential for the design and development of materials. The CALPHAD (Calculation of Phase Diagrams) technique has made it possible to calculate properties of multicomponent systems using databases of thermodynamic descriptions with models that were assessed from experimental data. A number of thermodynamic databases and softwares are available for the calculation of properties of multicomponent systems under the frame of ICME (Integrated Computational Material Engineering) or MGI (Materials Genome Initiative). This article briefly reviewed the past and present of the thermodynamic databases and softwares and discussed their future development combining our own research work.
\end{abstract}

Keywords: thermodynamic databases, softwares, CALPHAD, ICME or MGI

doi: $10.1360 / \mathrm{N} 032018-00258$ 